\title{
Sorafenib in the treatment of radioiodine-refractory differentiated thyroid cancer: a meta-analysis
}

\author{
Chen-Tian Shen*, Zhong-Ling Qiu* and Quan-Yong Luo
}

Department of Nuclear Medicine, Shanghai Jiao Tong University Affiliated Sixth People's Hospital, 600 Yishan Road, Shanghai 200233, People's Republic of China

*(C-T Shen and Z-L Qiu contributed equally to this work)

Correspondence should be addressed to Q-Y Luo

Email Iqyn@sh163.net

\begin{abstract}
The advent of biologically targeted agents and increased understanding of thyroid carcinogenesis have generated much interest in the development of biologically targeted therapeutic agents for thyroid cancer. Among them, sorafenib is the most commonly studied drug. The current meta-analysis was carried out to estimate the efficacy and safety of sorafenib administered in radioiodine-refractory differentiated thyroid cancer patients. An electronic search was conducted using PubMed/MEDLINE and EMBASE. Statistical analyses were carried out using either random-effects or fixed-effects models according to heterogeneity. All the statistical analyses were carried out using the Stata version 12.0 software. Seven eligible studies were identified. The final results indicated that $22 \%$ of the patients ( $95 \% \mathrm{Cl}: 15-28)$ achieved a partial response. Hand-foot syndrome, diarrhea, fatigue, rash, weight loss, and hypertension were the most frequently observed adverse effects (AEs) associated with sorafenib use and the incidence of these AEs (all grades) was $80 \%$ (95\% Cl: 68-91), 68\% (95\% Cl: 59-77), $67 \%(95 \% \mathrm{Cl}: 57-78), 66 \%(95 \% \mathrm{Cl}: 50-82), 52 \%(95 \% \mathrm{Cl}: 33-72)$, and $31 \%(95 \% \mathrm{Cl}: 21-42)$ respectively. Sixty-two percent ( $95 \% \mathrm{Cl}: 36-89)$ patients required dose reductions due to toxicity of sorafenib. As far as PR and AEs are concerned, the results of this meta-analysis indicate that sorafenib has a modest effect in patients with radioiodine-refractory differentiated thyroid cancer and the high incidence of AEs associated with this agent may affect the quality of patients' lives. Though the use of sorafenib in the treatment of radioiodinerefractory differentiated thyroid cancer is considered promising by most physicians working in this field, more effective agents with less toxicity and cost are still needed.
\end{abstract}
Key Words
- sorafenib
- tyrosine kinase inhibitors
- differentiated thyroid cancer
- meta-analysis
- molecular targeted therapy

Endocrine-Related Cancer (2014) 21, 253-261

\section{Introduction}

Thyroid carcinoma is the most common endocrine malignancy. While the incidence of most cancers is on the decline, the worldwide incidence of thyroid carcinoma is increasing in both men and women. It has been estimated that in 2013, 60220 patients will be diagnosed with thyroid carcinoma in the USA and about 1850 will die (Siegel et al. 2013). Differentiated thyroid carcinoma
(DTC) is the most common (95\%) subtype of thyroid carcinoma accounting for approximately $2 \%$ of all malignancies in the USA (Eheman et al. 2012). Due to the biological behavior of the tumor, the prognosis of DTC is excellent following conventional treatments based on adequate surgical management and radioactive iodine (RAI) ablation as well as TSH suppression (Dadu \& Cabanillas 2012). 
However, approximately $10-20 \%$ of DTC patients will develop distant metastases and about $50 \%$ of them will die within 10 years of diagnosis. It is estimated that $5 \%$ of DTC patients will lose ${ }^{131}$ I avidity and become resistant to RAI, which is a crucial tool for the treatment of DTC. This results in an aggressive behavior and a poor prognosis with long-term overall survival (OS) of only 10\% (Regalbuto et al. 2012).

The medical approach for the treatment of advanced or metastatic DTC refractory to conventional treatment was considered particularly challenging. Historically, the role of cytotoxic chemotherapy has been quite limited in these patients due to both limited need and efficacy when used (Sherman 2010). Recently, the knowledge of key mutational events in some genes (mainly focused on RET/PTC, RAS (NRAS, KRAS, HRAS), BRAF, and EGFR) has been promulgated widely. These mutations are considered to be connected to a signaling net that is responsible for the carcinogenesis and angiogenesis of thyroid cancer (Albarel et al. 2012). Moreover, many novel biological agents that target these mutations have been identified and studied, which brings hope to patients with thyroid cancer especially to those with radioiodine-refractory DTC. Among them, sorafenib is the most commonly studied drug.

Sorafenib is an orally active multi-tyrosine kinase inhibitor (TKI) targeting both cell-surface tyrosine kinase receptors and downstream intracellular serine/threonine kinases in the Ras/MAPK cascade. This drug affects tumor cell proliferation and angiogenesis and has recently been approved in the USA by the Food and Drug Administration for the treatment of renal cell and hepatocellular carcinomas (Wilhelm et al. 2006). It is the first compound capable of inhibiting RAF kinases and targeting a panel of tyrosine kinase receptors such as VEGF receptors 1-3 and PDGFRB as well as RET, c-KIT, and FLT3. These characteristics provide the possible molecular rationale for the use of sorafenib in the treatment of all subtypes of thyroid cancer (Duntas \& Bernardini 2010).

So far, several clinical trials have been conducted to evaluate the effectiveness and adverse effects (AEs) of sorafenib in patients with radioiodine-refractory DTC, and some reviews of novel targeted therapies available for patients with all types of thyroid cancer are currently being reported. However, to our knowledge, no metaanalysis has been carried out to estimate the efficacy and safety of sorafenib administered in radioiodine-refractory DTC patients. Therefore, the current meta-analysis attempted to analyze and combine the results of these clinical trials to increase the statistical power and improve the estimates of the effects.

\section{Materials and methods}

\section{Literature search for identifying related studies}

A search for human trials without language restrictions using the bibliographic databases PubMed/MEDLINE and EMBASE was conducted using the terms 'thyroid cancer', 'thyroid carcinoma', and 'radioiodine refractory' as well as the term 'sorafenib' and related pharmaceutical names. A list of articles was obtained through extensive crosschecking of the reference lists of all the retrieved articles. Unpublished data and conference proceedings were not included.

\section{Study selection}

Two reviewers (Z-L Q and C-T S) independently assessed the eligibility of each article. After screening all the titles and reading the abstracts, full texts of the selected articles were reviewed to determine their eligibility for inclusion in the study. Disagreement between reviewers was resolved by consensus. To be included in the meta-analysis: (1) studies had to use sorafenib; (2) studies had to record necessary data about therapy efficacy and safety; and (3) studies had to be carried out in patients with a diagnosis of radioiodinerefractory DTC. Exclusion criteria were as follows: (1) involvement of malignancy that was not thyroid cancer; (2) use of pharmaceuticals that were not biologically targeted agents; (3) animal and cell studies; and (4) letters, abstracts, reviews, case reports, editorials, and comments.

\section{Data extraction}

Data extraction was conducted independently by two investigators (C-T S and Z-L Q) according to the Preferred Reporting Items for Systematic Reviews and Meta-Analyses (PRISMA) statement (Moher et al. 2009). For each relevant study, collected data included the following: first author's name, year of publication, country, trial design, number of enrolled subjects, median age of enrolled subjects, gender composition, drug dose, median treatment duration, median partial response (PR), median stable disease (SD), median progression-free survival (PFS), and OS. To resolve disagreements between reviewers, a third reviewer (Q-Y L) assessed all the discrepant items.

To evaluate the toxicity of sorafenib, the authors calculated the number of events of the following AEs reported in the safety profile section: hand-foot syndrome, diarrhea, fatigue, rash, weight loss, and hypertension. When available, all-grade (1-4) and high-grade (3-4) events according to the Common Terminology

Published by Bioscientifica Ltd. 
Criteria for Adverse Events (CTCAE Version 3.0; National Cancer Institute, Bethesda, MD, USA) were included in the meta-analysis.

\section{Statistical analyses}

For each trial, the rate of PR and stability of disease, PFS, and main AEs were analyzed from the extracted data and 95\% CIs were derived. Heterogeneity analysis was carried out by calculating the $I^{2}$, which was interpreted as low (25\%), moderate (50\%), and high (75\%) (Moher et al. 2009). According to one review (Berlin 1995), the $I^{2}$ index assessed not only heterogeneity in a meta-analysis but also the extent of that heterogeneity. It is considered a more appropriate procedure than Dixon's $Q$ test for assessing whether there is true heterogeneity among the studies in a meta-analysis (Huedo-Medina et al. 2006). For the meta-analysis, both fixed-effects (weighted with inverse variance) and randomeffects models were considered (DerSimonian \& Laird 1986). A random-effects model was chosen when heterogeneity was $>50 \%$, while a fixed-effects model was chosen when heterogeneity was $<50 \%$. Publication bias was assessed using a standard funnel plot (Copas \& Shi 2000). Forest plots were sorted according to the first author's name, year, and country to illustrate the ratio of PR and SD, PFS, and incidence of AEs. All the statistical analyses were carried out using the Stata version 12.0 software (StataCorp. 4905 Lakeway Drive College Station, Texas, 77845, USA).

\section{Results}

\section{Study characteristics}

The literature search identified 189 potentially relevant articles. After screening titles and abstracts, 176 nonrelevant articles were excluded as being for other malignancies, treatment agents, review articles, case reports, abstracts presented at meetings, letters, and commentaries. Following a more detailed review, six articles were excluded because two combined sorafenib with other agents, two mainly referred to molecular mechanisms, two had a duplicate trial, and one included only anaplastic thyroid carcinoma (ATC) cases. The remaining seven trials involving 211 patients fulfilled our inclusion criteria (GuptaAbramson et al. 2008, Kloos et al. 2009, Ahmed et al. 2011, Chen et al. 2011, Capdevila et al. 2012, Schneider et al. 2012, Marotta et al. 2013). The process of study selection is shown as a flow chart in Fig. 1.

The baseline characteristics of each trial are given in Table 1 . These seven trials were published between 2008

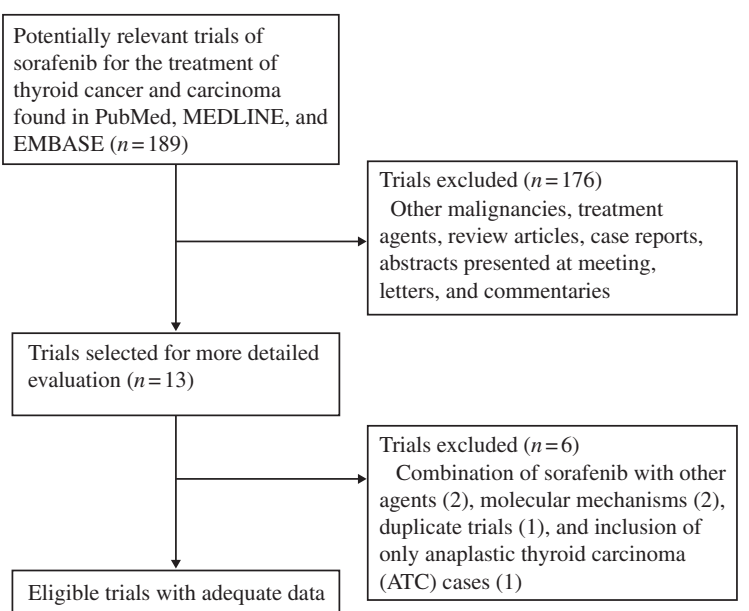

Figure 1

Flow chart of the study selection process.

and 2013, including two retrospective and five prospective trials. In all, 211 patients (115 men and 96 women, median age: $55-67$ years) were identified. All the selected trials involved patients with radioiodine-refractory DTC (four articles (Gupta-Abramson et al. 2008, Kloos et al. 2009, Ahmed et al. 2011, Capdevila et al. 2012) also included a small proportion of medullary thyroid carcinoma (MTC) and/or ATC cases and, which were excluded when analyzed). These trials were carried out in six countries: the USA, the UK, China, Italy, the Netherlands, and Spain. All the trials reported an Eastern Cooperative Oncology Group (ECOG) performance status $\leq 2$, except one trial, which included six patients whose ECOG status was 3 (Marotta et al. 2013). The dose of sorafenib administered was $400 \mathrm{mg}$ orally twice a day (PO b.i.d.) in all the trials, except in one trial, which used $200 \mathrm{mg}$ PO b.i.d. (Chen et al. 2011). The efficacy of each trial is summarized in Table 2, along with the evaluation index including PR, stability of disease, clinical benefits, and PFS and OS rates. High-grade (3-4) events reported in each trial are presented in Table 3.

\section{Efficacy of sorafenib in radioiodine-refractory DTC}

The occurrence PR of sorafenib in patients with radioiodine-refractory DTC ranged from 15 to $33 \%$, and no complete response (CR) was reported. SD ranged from 41 to $82 \%$, PFS ranged from 4.5 to 19.6 months, and OS ranged from 10 to 37.5 months. The PR, SD, and PFS were further meta-analyzed for all the radioiodine-refractory DTC patients and the results were 22\% (95\% CI: 15-28;

Published by Bioscientifica Ltd 
Table 1 Baseline characteristics of each trial

\begin{tabular}{|c|c|c|c|c|c|c|c|c|c|}
\hline References & Country & Trial design & $\begin{array}{c}\text { Dose } \\
(\mathrm{mg} \text { b.i.d) }\end{array}$ & $\begin{array}{l}\text { Number of } \\
\text { patients }\end{array}$ & Type & ECOG & $\begin{array}{l}\text { Patients } \\
(\mathrm{M}+\mathrm{F}) / \mathbf{n}\end{array}$ & $\begin{array}{c}\text { Median } \\
\text { age }(y)\end{array}$ & $\mathbf{T}(\mathrm{m})$ \\
\hline $\begin{array}{l}\text { Gupta-Abramson } \\
\text { et al. (2008) }\end{array}$ & USA & $\begin{array}{l}\text { Phase II: } \\
\quad \text { prospective }\end{array}$ & 400 & 30 & $\begin{array}{l}27 \text { DTC/1 } \\
\text { MTC/2 ATC }\end{array}$ & $0-1$ & $30(15+15)$ & 63 & 6.3 \\
\hline \multirow[t]{4}{*}{ Kloos et al. (2009) } & USA & $\begin{array}{l}\text { Phase II: } \\
\text { prospective }\end{array}$ & 400 & 56 & $\begin{array}{l}52 \mathrm{DTC} / 4 \text { ATC } \\
\text { Arm A }\end{array}$ & $--^{a}$ & $56(31+25)$ & $-^{a}$ & $--^{a}$ \\
\hline & & & & & $\begin{array}{l}19 \text { PTC } \\
\text { Arm B }\end{array}$ & & $19(11+8)$ & 67 & 14 \\
\hline & & & & & 22 PTC & & $22(10+12)$ & 56 & 10 \\
\hline & & & & & $\begin{array}{l}11 \mathrm{HTC} / \mathrm{FTC} \\
4 \mathrm{ATC}\end{array}$ & & $15(10+5)$ & 61 & $\begin{array}{l}8 \\
2\end{array}$ \\
\hline $\begin{array}{l}\text { Ahmed et al. } \\
\text { (2011) }\end{array}$ & UK & $\begin{array}{l}\text { Phase II: } \\
\quad \text { prospective }\end{array}$ & 400 & 34 & $\begin{array}{l}19 \mathrm{DTC} / 15 \\
\mathrm{MTC}\end{array}$ & $0-1$ & $34(19+15)$ & 55 & 16.5 \\
\hline Chen et al. (2011) & China & Prospective & 200 & 9 & PTC & $0-2$ & $9(7+2)$ & 57 & $-^{a}$ \\
\hline $\begin{array}{l}\text { Schneider et al. } \\
\text { (2012) }\end{array}$ & $\begin{array}{l}\text { The } \\
\text { Netherlands }\end{array}$ & $\begin{array}{l}\text { Phase II: } \\
\text { prospective }\end{array}$ & 400 & 31 & DTC & $0-2$ & $31(19+12)$ & 64 & 15 \\
\hline $\begin{array}{l}\text { Capdevila et al. } \\
\text { (2012) }\end{array}$ & Spain & Retrospective & 400 & 34 & $\begin{array}{l}16 \text { DTC/15 } \\
\text { MTC/3 ATC }\end{array}$ & $0-2$ & $34(16+18)$ & 57.3 & $-^{a}$ \\
\hline $\begin{array}{l}\text { Marotta et al. } \\
(2013)\end{array}$ & Italy & Retrospective & 400 & 17 & DTC & $0-3$ & $17(8+9)$ & 61 & - \\
\hline
\end{tabular}

b.i.d, twice a day; DTC, differentiated thyroid carcinoma; MTC, medullary thyroid carcinoma; ATC, anaplastic thyroid carcinoma; PTC, papillary thyroid carcinoma; HTC, Hürthle cell thyroid carcinoma; FTC, follicular thyroid carcinoma; ECOG, Eastern Cooperative Oncology Group; M, male; F, female; $n$, number; $y$, year; $T$, median treatment duration; $\mathrm{m}$, month.

${ }^{a}$ Not reported in the trial.

$P=0.000), \quad 52 \% \quad(95 \% \quad \mathrm{CI}: \quad 44-60 ; \quad P=0.000)$, and 12.4 months (95\% CI: $10.4-14.7 ; P=0.000)$ respectively (Fig. 2A, B, and C). All of them were pooled using the fixed-effects model according to their heterogeneity. OS was not meta-analyzed because three of the trials did not fulfill the OS criteria.

\section{Safety}

The most commonly reported AEs included hand-foot syndrome, diarrhea, and rash. Rare but severe AEs were observed mainly due to intracranial hemorrhage (Capdevila et al. 2012), cardiac arrest (Marotta et al. 2013), angioedema, small-cell lung cancer, carcinoma of the tongue (Schneider et al. 2012), and grade 5 event of sudden death (Kloos et al. 2009). Meta-analyses of patients with all-grade hand-foot syndrome, diarrhea, fatigue, rash, weight loss, and hypertension revealed that the incidence was 80\% (95\% CI: 68-91; $P=0.000), 68 \%$ (95\% CI: 59-77; $P=0.000), \quad 67 \%$ (95\% CI: 57-78; $P=0.000), 66 \%$ (95\% CI: 50-82; $P=0.000), 52 \%$ (95\% CI: $33-72 ; P=0.000)$, and $31 \%$ (95\% CI: $21-42 ; P=0.000)$

Table 2 Evaluation indexes reflecting the efficacy of sorafenib in each trial

\begin{tabular}{|c|c|c|c|c|c|c|c|}
\hline References & Patients $(n)$ & Type & $\mathbf{P R} \times \mathbf{n} / \%$ & $\mathbf{S D} \times \boldsymbol{n} / \%$ & $\begin{array}{c}\text { Clinical } \\
\text { benefits }(\%)\end{array}$ & PFS (months) & OS (months) \\
\hline $\begin{array}{l}\text { Gupta-Abramson et al. } \\
\text { (2008) }\end{array}$ & 27 & DTC & $7 / 26$ & $15 / 56$ & 81 & 19.6 & $-{ }^{a}$ \\
\hline \multirow[t]{4}{*}{ Kloos et al. (2009) } & 52 & 41 PTC & $6 / 15$ & $23 / 56$ & 71 & 15 & $-^{a}$ \\
\hline & & 33 PTC & $5 / 15$ & $19 / 57$ & 73 & 16 & 23 \\
\hline & & 8 PTC $^{b}$ & $1 / 13$ & $6 / 75$ & 88 & 10 & 37.5 \\
\hline & & $11 \mathrm{HTC}$ or FTC & $0 / 0$ & $9 / 82$ & 82 & 4.5 & 24.2 \\
\hline Ahmed et al. (2011) & 19 & DTC & $3 / 16$ & $13 / 68$ & 84 & $-^{a}$ & $-{ }^{a}$ \\
\hline Chen et al. (2011) & 9 & DTC & $3 / 33$ & $4 / 44$ & 77 & 9.8 & $-^{a}$ \\
\hline Schneider et al. (2012) & 31 & DTC & $8 / 31$ & $11 / 42$ & 73 & 18 & 34.5 \\
\hline Capdevila et al. (2012) & 16 & DTC & $3 / 19$ & $8 / 50$ & 69 & 13.3 & 23.6 \\
\hline Marotta et al. (2013) & 17 & DTC & $5 / 30$ & $7 / 41$ & 71 & 9 & 10 \\
\hline
\end{tabular}

n, number; DTC, differentiated thyroid carcinoma; PTC, papillary thyroid carcinoma; HTC, Hürthle cell thyroid carcinoma; FTC, follicular thyroid carcinoma; $\mathrm{PR}$, partial response; SD, stable disease; PFS, progression-free survival; OS, overall survival.

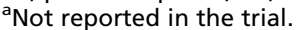

${ }^{b}$ Patients treated with chemotherapy before being treated with sorafenib. 
Table 3 Grade 3 AEs ( $>5 \%$ frequency) and grade 4 AEs of sorafenib reported in each trial

\begin{tabular}{|c|c|c|c|c|c|}
\hline References & High-grade AEs (\%) & $\begin{array}{c}\text { Dose reduction } \\
(n / \%)\end{array}$ & $\begin{array}{c}\text { Therapy } \\
\text { discontinuation } \\
\text { due to toxicities } \\
(n / \%)\end{array}$ & $\begin{array}{l}\text { Fatalities on } \\
\text { therapy }^{\mathrm{a}}(n / \%)\end{array}$ & $\begin{array}{l}\text { Adjustment of } \\
\text { thyroid hormone } \\
\text { requirements } \\
(n / \%)\end{array}$ \\
\hline $\begin{array}{l}\text { Gupta-Abramson et al. } \\
\text { (2008) }\end{array}$ & $\begin{array}{l}\text { Grades } 3-4^{\text {b}} \text { : hypertension (13), HFS } \\
\text { (10), rash (10), weight loss (10), } \\
\text { diarrhea (7), and elevated LFTs (7) }\end{array}$ & $14 / 47$ & $6 / 20$ & $1 / 3$ & $10 / 33$ \\
\hline Kloos et al. (2009) & $\begin{array}{l}\text { Grade 3: hand or foot pain (12), } \\
\text { arthralgia (11), fatigue (16), } \\
\text { HFS (7), musculoskeletal chest } \\
\text { pain (7), and asymptomatic } \\
\text { hyponatremia (5) } \\
\text { Grade 4: pericardial effusion and } \\
\text { reversible neutropenia }\end{array}$ & $29 / 52$ & $14 / 25$ & $24 / 43$ & $0 / 0$ \\
\hline Ahmed et al. (2011) & $\begin{array}{l}\text { Grade 3: HFS (44), other dermato- } \\
\text { logical diseases (6), infection (9), } \\
\text { fatigue (9), mucositis (9), } \\
\text { arthralgia (9), hypertension (6), } \\
\text { and drug hypersensitivity (9) } \\
\text { Grade 4: febrile neutropenia and } \\
\text { ruptured aortic graft }\end{array}$ & $28 / 82$ & $2 / 6$ & $2 / 6$ & $4 / 12$ \\
\hline Chen et al. (2011) & None & $0 / 0$ & $0 / 0$ & $1 / 11$ & $0 / 0$ \\
\hline Schneider et al. (2012) & $\begin{array}{l}\text { Grade 3: hypertension (16), HFS } \\
\text { (23), rash (16), mucositis (10), } \\
\text { weight loss (10), diarrhea (6), and } \\
\text { elevated LFTs (7) } \\
\text { Grade 4: myocardial infarction and } \\
\text { small-cell lung cancer }\end{array}$ & $18 / 58$ & $7 / 23$ & $1 / 3$ & $13 / 42$ \\
\hline Capdevila et al. (2012) & $\begin{array}{l}\text { Grade 3: HFS (20), diarrhea (15), } \\
\text { alopecia (12), rash (6), fatigue } \\
\text { (15), and anorexia (9) } \\
\text { Grade 4: HFS and elevated LFTs }\end{array}$ & $12 / 35$ & $0 / 0$ & $1 / 3$ & $0 / 0$ \\
\hline Marotta et al. (2013) & $\begin{array}{l}\text { HFS, etc. (details were not } \\
\text { reported) }\end{array}$ & $17 / 100$ & $2 / 12$ & $5 / 29$ & $13 / 76$ \\
\hline
\end{tabular}

AEs, adverse effects; TH, thyroid hormone; HFS, hand-foot syndrome; LFTs, liver function tests.

${ }^{\text {a } F a t a l i t i e s ~ o f ~ a l l ~ c a u s e s . ~}$

${ }^{{ }^{b}}$ High-grade AEs were not reported separately in the trial and only grade 3-4 AEs were reported.

respectively (Fig. 3A, B, C, D, E, and F). A meta-analysis was also carried out on the proportion of patients in six trials requiring dose reductions due to the toxicity of sorafenib (one trial was excluded because the initial dose used was already $200 \mathrm{mg}$ PO b.i.d. (Chen et al. 2011)) and the incidence was 62\% (95\% CI: 36-89; $P=0.000$ ) (Fig. 2D). All of them were pooled using the random-effects model, except diarrhea (which was pooled using the fixed-effects model instead), according to their heterogeneity. Other relative data such as the incidences of therapy discontinuation due to toxicities, fatalities on therapy, and adjustment of thyroid hormone requirements were also obtained and are presented in Table 3 .

\section{Discussion}

Despite many comprehensive and systematic reviews of the literature on novel targeted therapies available for patients with all types of thyroid cancer being reported recently (Brose et al. 2012, Busaidy \& Cabanillas 2012, Kojic et al. 2012, Perez et al. 2012, Anderson et al. 2013), to our knowledge, this is the first meta-analysis conducted to estimate the efficacy and safety of sorafenib administered in radioiodine-refractory DTC patients.

In the current meta-analysis, the pooled PR in patients with radioiodine-refractory DTC was $22 \%$. As has been mentioned above, many other TKIs have been studied in patients with radioiodine-refractory DTC mainly comprising sunitinib, motesanib, pazopanib, and vandetanib (Sherman et al. 2008, Bible et al. 2010, Carr et al. 2010, Leboulleux et al. 2012). Among them, pazopanib exhibited confirmed PR in 18 of the 37 patients (49\%) with metastatic, rapidly progressive, radioiodine-refractory differentiated thyroid cancer, with the likelihood of response lasting longer than 1 year calculated to be $66 \%$ (Bible et al. 2010). At the same time, several non-TKIs have

Published by Bioscientifica Ltd 
A

\begin{tabular}{|c|c|c|c|c|}
\hline Study & Year & \multicolumn{2}{|l|}{ Country } & PR (95\% Cl) \\
\hline Gupta-Abramson et al. & 2008 & USA & 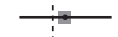 & $0.26(0.09,0.43)$ \\
\hline Kloos et al. & 2009 & USA & $\rightarrow$ & $0.15(0.04,0.26)$ \\
\hline Ahmed et al. & 2011 & UK & $\longrightarrow$ & $0.16(-0.00,0.32)$ \\
\hline Chen et al. & 2012 & China & + & $0.33(0.02,0.64)$ \\
\hline Schneider et al. & 2012 & Netherlands & & $0.31(0.15,0.47)$ \\
\hline Capdevila et al. & 2012 & Spain & $\longrightarrow$ & $0.19(-0.00,0.38)$ \\
\hline Marotta et al. & 2013 & Italy & 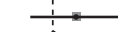 & $0.30(0.08,0.52)$ \\
\hline \multicolumn{3}{|c|}{ Overall $\left(I^{2}=0.0 \%, P=0.600\right)$} & & $0.22(0.15,0.28)$ \\
\hline
\end{tabular}

B

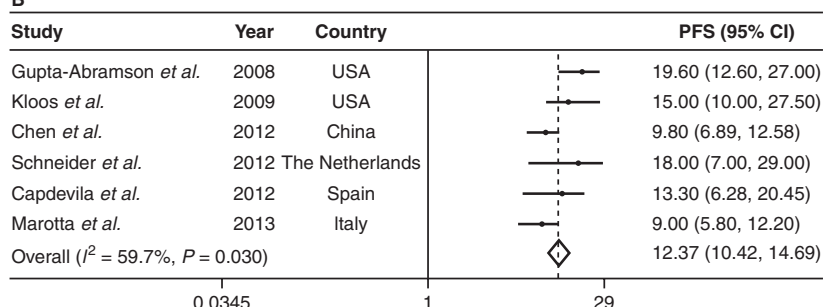

C

\begin{tabular}{|c|c|c|c|c|}
\hline Study & Year & Country & & SD\% (95\% Cl) \\
\hline Gupta-Abramson et al. & 2008 & USA & 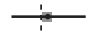 & $0.56(0.37,0.75)$ \\
\hline Kloos et al. & 2009 & USA & & $0.56(0.14,0.71)$ \\
\hline Ahmed et al. & 2011 & UK & & $0.68(0.47,0.89)$ \\
\hline Chen et al. & 2012 & China & & $0.44(0.12,0.76)$ \\
\hline Schneider et al. & 2012 & e Netherlands & & $0.42(0.25,0.59)$ \\
\hline Capdevila et al. & 2012 & Spain & & $0.50(0.26,0.74)$ \\
\hline Marotta et al. & 2013 & Italy & & $0.41(0.18,0.64)$ \\
\hline \multicolumn{3}{|c|}{ Overall $\left(I^{2}=0.0 \%, P=0.536\right)$} & & $0.52(0.44,0.60)$ \\
\hline
\end{tabular}

D

\begin{tabular}{|c|c|c|}
\hline Study & ES $(95 \% \mathrm{Cl})$ & Weight ${ }^{\circ}$ \\
\hline Gupta-Abramson et al. & $0.47(0.29,0.65)$ & 5) 16.22 \\
\hline Kloos et al. & $0.52(0.39,0.65)$ & 5) 16.80 \\
\hline Ahmed et al. & $0.79(0.65,0.93)$ & 3) 16.73 \\
\hline Schneider et al. & $0.58(0.41,0.75)$ & 5) 16.29 \\
\hline Capdevila et al. & $0.35(0.19,0.51)$ & l) 16.46 \\
\hline Marotta et al. & . $1.00(0.98,1.01)$ & 17.51 \\
\hline Overall $\left(I^{2}=97.1 \%, P=0.000\right)$ & $0.62(0.36,0.89)$ & 100.0 \\
\hline
\end{tabular}

Figure 2

Forest plots illustrating the efficacy of sorafenib in patients with radioiodine-refractory DTC. (A) the ratio of PR; (B) PFS; (C) the ratio of SD; and (D) ES (the incidence of dose reduction due to the adverse effects of sorafenib).

been studied as well, mainly comprising rosiglitazone, vorinostat, thalidomide, celecoxib, and selumetinib (Mrozek et al. 2006, Ain et al. 2007, Kebebew et al. 2009, Woyach et al. 2009, Ho et al. 2013). Though most of them exhibited modest efficacy, selumetinib, a MAPK kinase (MEK) 1 and MEK 2 inhibitor, increased the uptake of iodine- 124 in 12 of the 20 patients (60\%). Of these 12 patients, eight reached the dosimetry threshold for radioiodine therapy (five had confirmed partial responses and three had stable disease). All the patients exhibited decreases in serum thyroglobulin levels (mean reduction: Printed in Great Britain
$\underline{A}$

\begin{tabular}{|c|c|c|}
\hline Study & HFS (95\% Cl) & Weight (\%) \\
\hline Gupta-Abramson et al. & $=0.93(0.84,1.02)$ & 24.24 \\
\hline Ahmed et al. & $-\quad 0.79(0.66,0.93)$ & 20.38 \\
\hline Marotta et al. & $-0.88(0.73,1.03)$ & 18.84 \\
\hline Schneider et al. & - $\quad 0.71(0.55,0.87)$ & 18.41 \\
\hline Capdevila et al. & $0.62(0.45,0.78)$ & 18.13 \\
\hline Overall $\left(I^{2}=72.2 \%, P=0.006\right)$ & $0.80(0.68,0.91)$ & 100.00 \\
\hline-1.03 & 1.03 & \\
\hline
\end{tabular}

B

\begin{tabular}{|c|c|c|c|}
\hline Study & & Rash $(95 \%$ Cl) & Weight (\%) \\
\hline Gupta-Abramson et al. & $\stackrel{+}{i}$ & $0.80(0.66,0.94)$ & 16.16 \\
\hline Kloos et al. & $\frac{1}{-1}$ & $0.79(0.68,0.89)$ & 28.70 \\
\hline Ahmed et al. & $\rightarrow$ & $0.88(0.77,0.99)$ & 28.16 \\
\hline Chen et al. & $\div$ & $0.44(0.12,0.77)$ & 3.14 \\
\hline Schneider et al. & $\longrightarrow$ & $0.58(0.41,0.76)$ & 11.00 \\
\hline Capdevila et al. & $\longrightarrow$ & $0.35(0.19,0.51)$ & 12.83 \\
\hline Overall $\left(I^{2}=86.2 \%, P=0.000\right)$ & $\diamond$ & $0.66(0.50,0.82)$ & 100.00 \\
\hline-0.99 & & 99 & \\
\hline
\end{tabular}

C

\begin{tabular}{|c|c|c|}
\hline Study & Diarrhea $(95 \% \mathrm{Cl})$ & Weight (\%) \\
\hline Gupta-Abramson et al. & $-0.80(0.66 .0 .94)$ & 17.18 \\
\hline Kloos et al. & $0.75(0.64,0.86)$ & 20.83 \\
\hline Ahmed et al. & $0.76(0.62,0.91)$ & 17.25 \\
\hline Chen et al. & - $\quad 0.44(0.12,0.77)$ & 5.82 \\
\hline Marotta et al. & $0.65(0.42,0.88)$ & 10.04 \\
\hline Schneider et al. & $0.52(0.34,0.69)$ & 13.85 \\
\hline Capdevila et al. & $0.62(0.45,0.78)$ & 15.04 \\
\hline Overall $\left(I^{2}=46.3 \%, P=0.083\right)$ & $0.68(0.59,0.77)$ & 100.00 \\
\hline
\end{tabular}

D

\begin{tabular}{|c|c|c|c|}
\hline Study & & Fatigue $(95 \% \mathrm{Cl})$ & Weight (\%) \\
\hline Gupta-Abramson et al. & $\longrightarrow$ & $0.63(0.46,0.81)$ & 17.21 \\
\hline Kloos et al. & & $0.82(0.72,0.92)$ & 25.22 \\
\hline Ahmed et al. & $\longrightarrow+$ & $0.59(0.42,0.75)$ & 17.89 \\
\hline Chen et al. & & $-0.67(0.36,0.97)$ & 8.39 \\
\hline Marotta et al. & & $0.71(0.49,0.93)$ & 13.54 \\
\hline Capdevila et al. & $\longrightarrow+1$ & $0.56(0.39,0.73)$ & 17.75 \\
\hline Overall $\left(I^{2}=53.3 \%, P=0.057\right)$ & 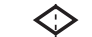 & $0.67(0.57,0.78)$ & 100.00 \\
\hline
\end{tabular}

E

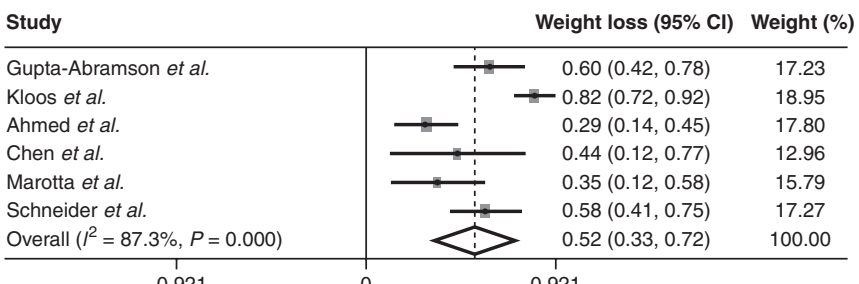

F

Study Hypertension $(95 \% \mathrm{Cl})$ Weight $(\%)$

\begin{tabular}{l|lll}
\hline Gupta-Abramson et al. & & $0.43(0.26,0.61)$ & 15.67
\end{tabular}

\begin{tabular}{l|rr} 
Gupta-Abramson et al. & $0.43(0.26,0.61)$ & 15.67 \\
Kloos et al. & $0.30,0.56)$ & 19.63
\end{tabular}

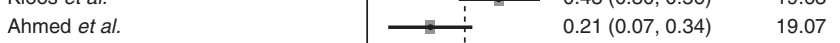

\begin{tabular}{lllll} 
Chen et al. & & & & \\
\cline { 2 - 2 } & & $0.22(-0.05,0.49)$ & 9.91
\end{tabular}

\begin{tabular}{l|lll} 
Schneider et al. & & $0.42(0.25,0.59)$ & 15.95
\end{tabular}

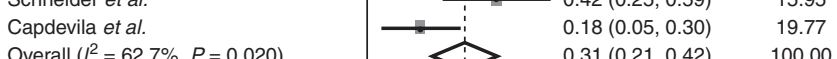

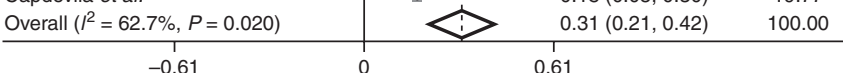

Figure 3

Forest plots illustrating the adverse effects associated with sorafenib. (A) HFS;

(B) rash; (C) diarrhea; (D) fatigue; (E) weight loss; and (F) hypertension. 
$89 \%$ ), and no toxic effects of grade 3 or higher attributable by the investigators to selumetinib were observed in this trial (Ho et al. 2013).

However, currently, a phase III randomized doubleblind, multicenter trial evaluating the safety and efficacy of sorafenib ( $400 \mathrm{mg}$ b.i.d.) vs placebo in patients with locally advanced or metastatic RAI-refractory DTC is underway (DECISION; ClinicalTrials.gov identifier: NCT00984282). The primary results of the trial were presented in the 2013 meeting of the American Society of Clinical Oncology Annual Meeting in Chicago, IL, which highlighted that the PFS in the sorafenib arm was significantly improved by 5 months compared with that in the placebo arm (Brose et al. 2013). Dose modifications due to AEs were more common with sorafenib (77.8\%) than with placebo (30.1\%), $18.8 \%$ of the patients discontinued sorafenib therapy due to AEs, and the most frequent serious AEs were secondary malignancies, occurring in $4.3 \%$ of the patients receiving sorafenib therapy in this trial (http://am. asco.org/sorafenib-shows-benefit-refractory-differentiatedthyroid-cancer, 30 October 2013).

The AEs (all grades) of sorafenib analyzed in the current study included hand-foot syndrome, diarrhea, fatigue, rash, weight loss, and hypertension. Grade 3 and 4 AEs mostly consisted of hand-foot syndrome, diarrhea, hypertension, rash, elevated abnormal liver function tests (LFTs), pericardial effusion, and neutropenia. Because of the limited data, the high-grade AEs were not meta-analyzed. Other rare but severe AEs associated with sorafenib such as eruptive keratoacanthoma-type squamous-cell carcinomas have also been reported (Smith et al. 2009).

Several factors had to be considered in the current meta-analysis. First, all the eligible trials lacked randomization and blinding, which might have resulted in an overestimation of the effects. Secondly, the inclusion criteria for each trial were different especially with regard to disease status at entry and previous therapies allowed. Five (Gupta-Abramson et al. 2008, Kloos et al. 2009, Chen et al. 2011, Capdevila et al. 2012, Schneider et al. 2012) of the seven eligible studies enrolled patients who had evidence of disease progression by the Response Evaluation Criteria in Solid Tumors in the 12 months preceding the initiation of treatment, while one (Ahmed et al. 2011) had evidence in the preceding 18 months and the other (Marotta et al. 2013) in the preceding 6 months. Five (Gupta-Abramson et al. 2008, Kloos et al. 2009, Ahmed et al. 2011, Capdevila et al. 2012, Marotta et al. 2013) of the seven eligible studies enrolled patients who had received prior chemotherapy. All the patients had not received prior therapy with TKIs, except two who had received prior therapy with investigational agents (no specific agents available) and one who had received therapy with a TKI, namely sunitinib (Gupta-Abramson et al. 2008, Capdevila et al. 2012). These differences in the aspects of inclusion criteria of the eligible studies may increase the heterogeneity. However, considering the fact that almost all the subjects had evidence of disease progression and the proportion of subjects who had received prior chemotherapy or biological therapies was relatively small, it can be assumed that this heterogeneity will not affect the effects greatly. Thirdly, the current meta-analysis was not based on individual patient data, another possible cause for the overestimation of the treatment effects. Finally, as has been mentioned above, there were some disagreements among the authors during the current meta-analysis, which were mainly focused on the eligibility of the trails. The eligibility of Chen's paper (Chen et al. 2011) was discussed by CT Shen and ZL Qiu because of its heterogeneity in drug dose and the relatively small number of subjects included. As a result, QY Luo resolved the disagreement and decided to include this trial in the meta-analysis. Though the drug dose used in this trial was $200 \mathrm{mg}$ b.i.d., the other trials had a great proportion of dose reduction, so this may actually decrease the heterogeneity. On the other hand, when dose reduction in the eligible trials was meta-analyzed, this trial was excluded because it had already administrated a relatively small dose. The authors actually did not set a limitation to the smallest number of subjects included in each trial, so nine patients were acceptable. The disagreement about prior therapies of the enrolled patients in all the eligible studies has already been discussed above.

In conclusion, as far as PR and AEs are concerned, the results of this meta-analysis indicate that sorafenib has a modest effect in patients with radioiodine-refractory differentiated thyroid cancer and the high incidence of AEs associated with sorafenib may affect the quality of patients' lives. Though the use of this TKI in the treatment of radioiodine-refractory differentiated thyroid cancer is considered promising by many physicians, more effective agents with less toxicity and cost are still needed.

Declaration of interest

The authors declare that there is no conflict of interest that could be perceived as prejudicing the impartiality of the research reported.

\section{Funding}

This research did not receive any specific grant from any funding agency in the public, commercial or not-for-profit sector.

Published by Bioscientifica Ltd. 


\section{Author contribution statement}

Z-L Qiu and C-T Shen independently assessed the eligibility of each article; C-T Shen and Z-L Qiu independently conducted data extraction according to the PRISMA statement; C-T Shen and Z-L Qiu independently carried out the statistical analyses; Q-Y Luo assessed all the discrepant items to resolve disagreements between C-T Shen and Z-L Qiu; and C-T Shen and Z-L Qiu wrote the paper, supervised by Q-Y Luo.

\section{References}

Ahmed M, Barbachano Y, Riddell A, Hickey J, Newbold KL, Viros A, Harrington KJ, Marais R \& Nutting CM 2011 Analysis of the efficacy and toxicity of sorafenib in thyroid cancer: a phase II study in a UK based population. European Journal of Endocrinology 165 315-322. (doi:10. 1530/EJE-11-0129)

Ain KB, Lee C \& Williams KD 2007 Phase II trial of thalidomide for therapy of radioiodine-unresponsive and rapidly progressive thyroid carcinomas. Thyroid 17 663-670. (doi:10.1089/thy.2006.0289)

Albarel F, Conte-Devolx B \& Oliver C 2012 From nodule to differentiated thyroid carcinoma: contributions of molecular analysis in 2012. Annales d'Endocrinologie 73 155-164. (doi:10.1016/j.ando.2012.03.002)

Anderson RT, Linnehan JE, Tongbram V, Keating K \& Wirth LJ 2013 Clinical, safety, and economic evidence in radioactive iodinerefractory differentiated thyroid cancer: a systematic literature review. Thyroid 23 392-407. (doi:10.1089/thy.2012.0520)

Berlin JA 1995 Invited commentary: benefits of heterogeneity in metaanalysis of data from epidemiologic studies. American Journal of Epidemiology 142 383-387.

Bible KC, Suman VJ, Molina JR, Smallridge RC, Maples WJ, Menefee ME, Rubin J, Sideras K, Morris JC III, McIver B et al. 2010 Efficacy of pazopanib in progressive, radioiodine-refractory, metastatic differentiated thyroid cancers: Results of a phase 2 consortium study. Lancet Oncology 11 962-972. (doi:10.1016/S1470-2045(10)70203-5)

Brose MS, Smit J, Capdevila J, Elisei R, Nutting C, Pitoia F, Robinson B, Schlumberger M, Shong YK \& Takami H 2012 Regional approaches to the management of patients with advanced, radioactive iodinerefractory differentiated thyroid carcinoma. Expert Review of Anticancer Therapy 12 1137-1147. (doi:10.1586/era.12.96)

Brose MS, Nutting CM, Jarzab B, Elisei R, Siena S, Bastholt L, de la Fouchardiere C, Pacini F, Paschke R, Shong YK et al. 2013 Sorafenib in locally advanced or metastatic patients with radioactive iodinerefractory differentiated thyroid cancer: the phase III DECISION trial. Journal of Clinical Oncology 31 (supplement; abstract 4).

Busaidy NL \& Cabanillas ME 2012 Differentiated thyroid cancer: management of patients with radioiodine nonresponsive disease. Journal of Thyroid Research 2012 618985. (doi:10.1155/2012/618985)

Capdevila J, Iglesias L, Halperin I, Segura A, Martínez-Trufero J, Vaz MÁ, Corral J, Obiols G, Grande E, Grau JJ et al. 2012 Sorafenib in metastatic thyroid cancer. Endocrine-Related Cancer 19 209-216. (doi:10.1530/ ERC-11-0351)

Carr LL, Mankoff DA, Goulart BH, Eaton KD, Capell PT, Kell EM, Bauman JE \& Martins RG 2010 Phase II study of daily sunitinib in FDG-PET-positive, iodine-refractory differentiated thyroid cancer and metastatic medullary carcinoma of the thyroid with functional imaging correlation. Clinical Cancer Research 16 5260-5268. (doi:10.1158/1078-0432.CCR-10-0994)

Chen L, Shen Y, Luo Q, Yu Y, Lu H \& Zhu R 2011 Response to sorafenib at a low dose in patients with radioiodine-refractory pulmonary metastases from papillary thyroid carcinoma. Thyroid 21 119-124. (doi:10.1089/ thy.2010.0199)

Copas J \& Shi JQ 2000 Meta-analysis, funnel plots and sensitivity analysis. Biostatistics 1 247-262. (doi:10.1093/biostatistics/1.3.247)

Dadu R \& Cabanillas ME 2012 Optimizing therapy for radioactive iodinerefractory differentiated thyroid cancer: current state of the art and future directions. Minerva Endocrinologica 37 335-356.
DerSimonian R \& Laird N 1986 Meta-analysis in clinical trials. Controlled Clinical Trials 7 177-188. (doi:10.1016/0197-2456(86)90046-2)

Duntas LH \& Bernardini R 2010 Sorafenib: rays of hope in thyroid cancer. Thyroid 20 1351-1358. (doi:10.1089/thy.2010.0056)

Eheman C, Henley SJ, Ballard-Barbash R, Jacobs EJ, Schymura MJ, Noone AM, Pan L, Anderson RN, Fulton JE, Kohler BA et al. 2012 Annual Report to the Nation on the status of cancer, 1975-2008, featuring cancers associated with excess weight and lack of sufficient physical activity. Cancer 118 2338-2366. (doi:10.1002/cncr.27514)

Gupta-Abramson V, Troxel AB, Nellore A, Puttaswamy K, Redlinger M, Ransone K, Mandel SJ, Flaherty KT, Loevner LA, O'Dwyer PJ et al. 2008 Phase II trial of sorafenib in advanced thyroid cancer. Journal of Clinical Oncology 26 4714-4719. (doi:10.1200/JCO.2008.16.3279)

Ho AL, Grewal RK, Leboeuf R, Sherman EJ, Pfister DG, Deandreis D, Pentlow KS, Zanzonico PB, Haque S, Gavane S et al. 2013 Selumetinib-enhanced radioiodine uptake in advanced thyroid cancer. New England Journal of Medicine 368 623-632. (doi:10.1056/ NEJMoa1209288)

Huedo-Medina TB, Sánchez-Meca J, Marín-Martínez F \& Botella J 2006 Assessing heterogeneity in meta-analysis: Q statistic or $I^{2}$ index? Psychological Methods 11 193-206. (doi:10.1037/1082-989X.11.2.193)

Kebebew E, Lindsay S, Clark OH, Woeber KA, Hawkins R \& Greenspan FS 2009 Results of rosiglitazone therapy in patients with thyroglobulinpositive and radioiodine-negative advanced differentiated thyroid cancer. Thyroid 19 953-956. (doi:10.1089/thy.2008.0371)

Kloos RT, Ringel MD, Knopp MV, Hall NC, King M, Stevens R, Liang J, Wakely PE Jr, Vasko VV, Saji M et al. 2009 Phase II trial of sorafenib in metastatic thyroid cancer. Journal of Clinical Oncology 27 1675-1684. (doi:10.1200/JCO.2008.18.2717)

Kojic KL, Kojic SL \& Wiseman SM 2012 Differentiated thyroid cancers: a comprehensive review of novel targeted therapies. Expert Review of Anticancer Therapy 12 345-357. (doi:10.1586/era.12.8)

Leboulleux S, Bastholt L, Krause T, de la Fouchardiere C, Tennvall J, Awada A, Gómez JM, Bonichon F, Leenhardt L, Soufflet C et al. 2012 Vandetanib in locally advanced or metastatic differentiated thyroid cancer: a randomised, double-blind, phase 2 trial. Lancet Oncology 13 897-905. (doi:10.1016/S1470-2045(12)70335-2)

Marotta V, Ramundo V, Camera L, Del Prete M, Fonti R, Esposito R, Palmieri G, Salvatore M, Vitale M, Colao A et al. 2013 Sorafenib in advanced iodine-refractory differentiated thyroid cancer: efficacy, safety and exploratory analysis of role of serum thyroglobulin and FDG-PET. Clinical Endocrinology 78 760-767. (doi:10.1111/ cen.12057)

Moher D, Liberati A, Tetzlaff J \& Altman DG 2009 Preferred Reporting Items for Systematic Reviews and Meta-Analyses: the PRISMA statement. Journal of Clinical Epidemiology 62 1006-1012. (doi:10.1016/j.jclinepi. 2009.06.005)

Mrozek E, Kloos RT, Ringel MD, Kresty L, Snider P, Arbogast D, Kies M, Munden R, Busaidy N, Klein MJ et al. 2006 Phase II study of celecoxib in metastatic differentiated thyroid carcinoma. Journal of Clinical Endocrinology and Metabolism 91 2201-2204. (doi:10.1210/jc.2005-2498)

Perez CA, Arango BA, Velez M, Raez LE \& Santos ES 2012 Emerging role of multikinase inhibitors for refractory thyroid cancer. Biologics 6 257-265. (doi:10.2147/BTT.S24465)

Regalbuto C, Frasca F, Pellegriti G, Malandrino P, Marturano I, Di Carlo I \& Pezzino V 2012 Update on thyroid cancer treatment. Future Oncology 8 1331-1348. (doi:10.2217/fon.12.123)

Schneider TC, Abdulrahman RM, Corssmit EP, Morreau H, Smit WA \& Kapiteijn E 2012 Long-term analysis of the efficacy and tolerability of sorafenib in advanced radio-iodine refractory differentiated thyroid carcinoma: final results of a phase II trial. European Journal of Endocrinology 167 643-650. (doi:10.1530/EJE-12-0405)

Sherman SI 2010 Cytotoxic chemotherapy for differentiated thyroid carcinoma. Clinical Oncology 22 464-468. (doi:10.1016/ j.clon.2010.03.014) 
Sherman SI, Wirth LJ, Droz JP, Hofmann M, Bastholt L, Martins RG, Licitra L, Eschenberg MJ, Sun YN, Juan T et al. 2008 Motesanib diphosphate in progressive differentiated thyroid cancer. New England Journal of Medicine 359 31-42. (doi:10.1056/NEJMoa075853)

Siegel R, Naishadham D \& Jemal A 2013 Cancer statistics, 2013. CA: A Cancer Journal for Clinicians 63 11-30. (doi:10.3322/caac.21166)

Smith KJ, Haley H, Hamza S \& Skelton HG 2009 Eruptive keratoacanthomatype squamous cell carcinomas in patients taking sorafenib for the treatment of solid tumors. Dermatologic Surgery 35 1766-1770. (doi:10.1111/j.1524-4725.2009.01289.x)
Wilhelm S, Carter C, Lynch M, Lowinger T, Dumas J, Smith RA, Schwartz B, Simantov R \& Kelley S 2006 Discovery and development of sorafenib: a multikinase inhibitor for treating cancer. Nature Reviews. Drug Discovery 5 835-844. (doi:10.1038/nrd2130)

Woyach JA, Kloos RT, Ringel MD, Arbogast D, Collamore M, Zwiebel JA, Grever M, Villalona-Calero M \& Shah MH 2009 Lack of therapeutic effect of the histone deacetylase inhibitor vorinostat in patients with metastatic radioiodine-refractory thyroid carcinoma. Journal of Clinical Endocrinology and Metabolism 94 164-170. (doi:10.1210/ jc.2008-1631)

Received in final form 1 December 2013

Accepted 2 December 2013

Made available online as an Accepted Preprint

3 December 2013
Published by Bioscientifica Ltd. 